\title{
Contribuição ao conhecimento da biologia reprodutiva de Molossus molossus Pallas, 1766) (Chiroptera, Molossidae)
}

\author{
Marta Elena Fabián 1, 2 \\ Rosane Vera Marques ${ }^{2}$
}

\begin{abstract}
Study of 179 specimens of Molossus molossus in Ceará, Brazil, showed morphological and functional ovarian asymmetry in adult females, with the right side more developed. Blastocyst implantation occurred also in the right uterine horn.

Active male testicles were bigger $(x=5,38 \mathrm{~mm})$ than inactive ones $(x=3,25$ $\mathrm{mm})$.

Pregnancy with subsequent offspring was found in March-April and November, during the wet season.
\end{abstract}

\section{INTRODUÇÃO}

Os morcegos da região neotropical apresentam padrão reprodutivo sazonal (FLEMING at al., 1972; WILSON, 1979). Segundo RACEY (1982) a reprodução estaria associada à disponibilidade de alimento e a fatores ambientais como temperatura, fotoperíodo e pluviosidade.

Espécies monoestras da família Molossidae têm o nascimento dos fillhotes associado à primavera, enquanto algumas espécies dos gêneros Molussos e Eumops são poliestras, podendo apresentar um estro pos-parto. Em todos os casos, os molossídeos geram apenas um filhote por gestação (CARTE, 1970).

Diversos autores têm estudado aspectos da biologia reprodutiva, principalmente de espécies de zonas temperadas e verificaram a diversidade de padróes que uma mesma espécie pode apresentar sob diferentes condições ambientais ou geograficas. Segundo TADDEI (1980) os fatores que regulam a atividade reprodutiva dos morcegos, não são totalmente conhecidos $\mathrm{e}$, com respeito às espécies tropicais, podem ser apenas estimados.

No Brasil, alguns trabalhos mencionam a presença de fêmeas grávidas ou a ocorrência de filhotes em determinadas épocas do ano (PERACCHI, 1968; TADDEI et al., 1976; SANTOS, 1978; BORNE, 1985). Outros são mais específicos, como os de TADDEI (1976) referindo-se à reprodução de espécies de Phyllostomidae no Estado de São Paulo, WILLIG (1983, 1985a, 1985b) sobre espécies da caatinga e cerrado do Nordeste do Brasil e MARQUES (1986) sobre Molossus ater da Amazônia.

1. Museu de Ciências Naturais da FZRGS. Caixa Postal,1188. 90610 Porto Alegre - RS.

2. Bolsista do CNPq. 
Revta bras. Zool.

41

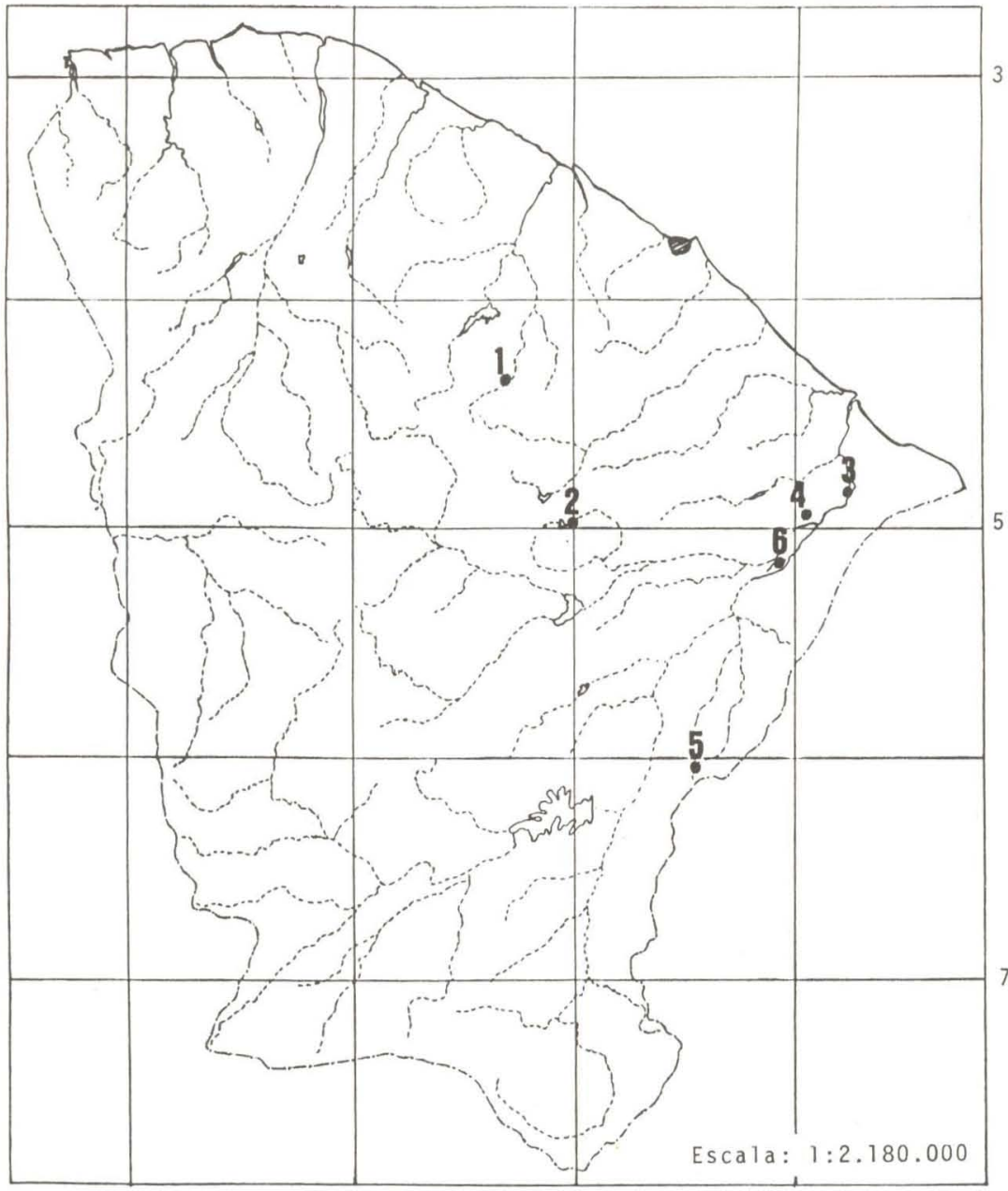

Fig. 1. Mapa do Estado do Ceará, Brasil, com indicação dos pontos de coleta: 1, Caninde; 2, Quixadá; 3, Jaguaruana; 4, Russas; 5, Pereiro; 6, Limoeiro do Norte.

O presente trabalho visa ampliar os conhecimentos sobre a reprodução de Molussus molussus, tendo sido realizado no período de junho de 1983 a novembro de 1984, nos municípios cearenses de Canindé, Quixadá, Jaguaruana, Russas, Limoeiro do Norte e Pereiro (Fig. 1). É importante constatar que, nas áreas estudadas, o período das chuvas estende-se, aproximadamente, de fevereiro a junho, sendo os demais meses do ano considerados secos, com exceção de novembro, quando podem ocorrer algumas chuvas esporádicas de pouca intensidade. 


\section{MATERIAL E MÉTODOS}

Fora examinados 179 exemplares de Molossus molossus. Deste total selecionaram-se 71 fêmeas e 53 machos adultos para estudo histológico das gônodas. O material foi fixado em formol a $10 \%$. Os exemplares coletados foram classificados em: machos adultos, machos jovens, fêmeas adultas não grávidas, fêmeas adultas grávidas, fêmeas jovens e filhotes.

Avaliou-se o aspecto morfológico dos testículos, efetuando-se as respectivas medidas, as quais foram analisadas estatisticamente através do teste $\mathbf{t}$ para amostras independentes, variâncias diferentes e para número de informações diferentes (SNEDECOR \& COCHRAN, 1967). Com relação às fêmeas, anotou-se o aspecto mor fológico do útero, trompas e ovários e posição do feto, no caso de fêmeas grávidas.

Os cortes histologicos das gônodas apresentaram a espessura de $7 \mu$ m e para sua coloração utilizou-se hematoxilina-eosina.

Os desenhos das gônodas foram efetuados mediante a utilização de câmara clara, acoplada a microscópio estereoscópico, em aumento de $10 \times 0.8$.

Os exemplares coletados estão devidamente registrados e depositados na coleção mastozoológica do Museu de Ciências Naturais da Fundação Zoobotânica do Rio Grande do Sul.

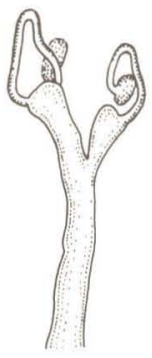

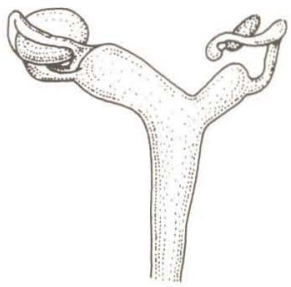

3

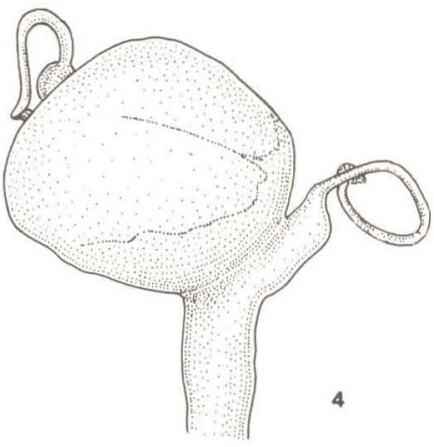

$10 \mathrm{~mm}$

Figs. 2-4. Molossus molossus. 2, Ovários e útero de fêmea jovem; 3, Ovários e útero de fêmea adulta; 4, Ovários e útero de fêmea grávida.

\section{RESULTADOS}

As fêmeas de Molussus molussus apresentam útero bicorne. Nos exemplares jovens, os cornos uterinos são aproximadamente do mesmo tamanho. Nas fêmeas adultas há grande diferença de tamanho entre os mesmos, sendo o corno direito 


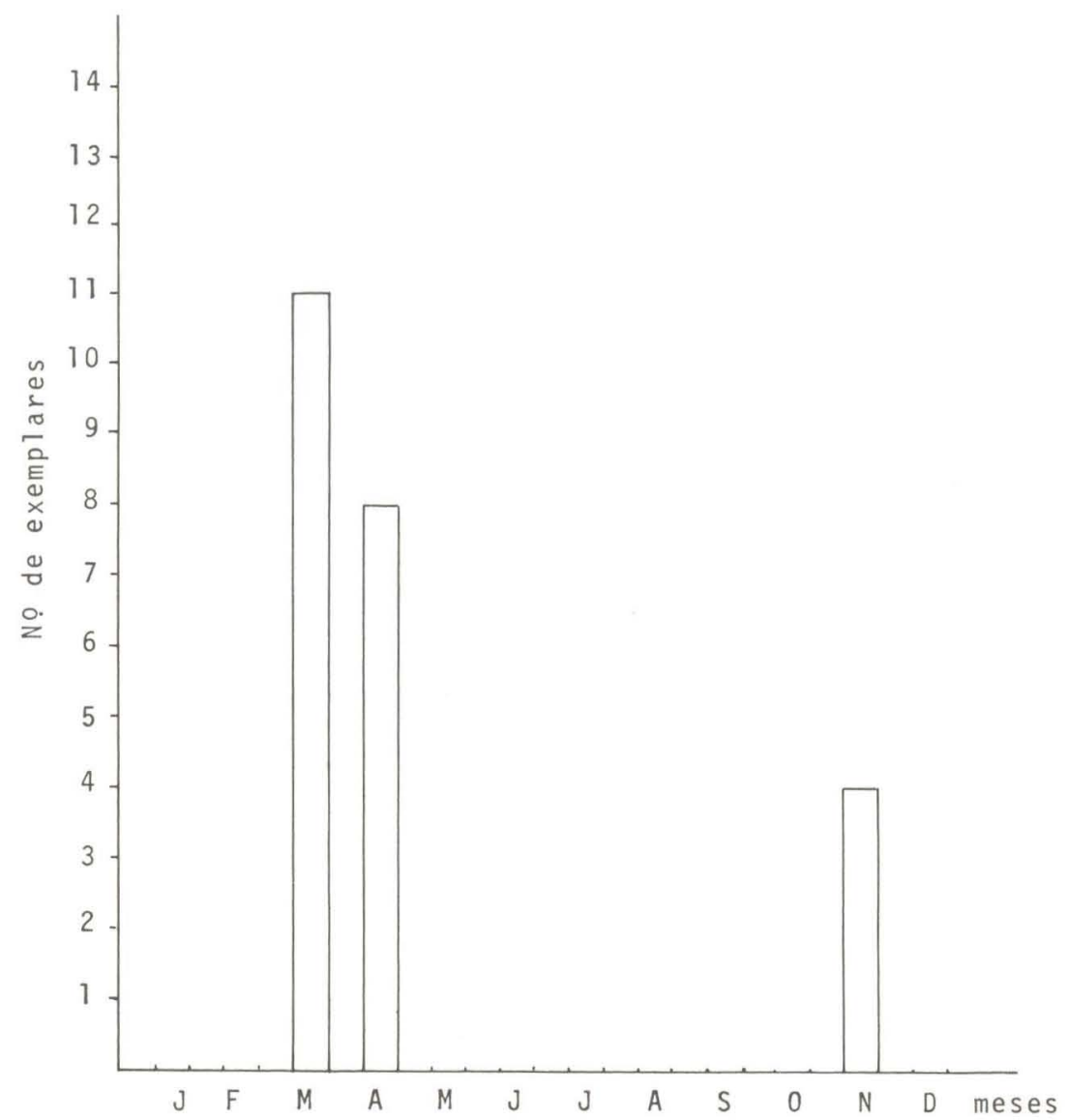

Fig. 5. Relação entre o número de fêmeas grávidas de Molossus molossus e os meses do ano.

mais desenvolvido que o esquerdo. Esta assimetria verifica-se também no tamanho dos ovários, onde o direito é visivelmente maior do que o esquerdo. As características morfológicas dos ovários e útero estão representadas nas figuras 2 a 4 .

Através do estudo microscópico verificou-se que, em $M$. molossus o ovário esquerdo contém apenas folículos primários e secundários. $\mathrm{O}$ desenvolvimento de folículos vesiculares assim como de corpo lúteo foi observado apenas no ovário direito. Nos dois ovários, os folículos distribuem-se junto ao córtex. A medida que os folículos primários vão se desenvolvendo em secundários e vesiculares, crescem em volume passando a ocupar a proção mais central do ovário. Normalmente os folículos são mono-ovulados, apesar de ter-se observado um folículo com dois ovócitos, em um único exemplar. As fêmeas grávidas apresentam um corpo lúteo bem 
desenvolvido no ovário direito; apenas num exemplar foram observados dois corpos lúteos concomitantemente.

A implantação de blastocisto ocorreu sempre no corno direito do útero. $\mathrm{O}$ maior desenvolvimento do lado direito permanece, mesmo após o período de gravidez ter sido concluido. Estas características demonstram uma assimetria não só morfológica como também funcional.

A poliestria parece bem caracterizada pela ocorrência de fêmeas grávidas nos meses de março-abril e novembro (Fig. 5). As fêmeas grávidas coletadas no mês de novembro eram jovens, com útero de tamanho muito reduzido. A presença de filhotes recém-nascidos ocorreu também nos meses de março-abril e novembro. Em M. molossus apenas um filhote se desenvolve por gestação. O período de nascimentos coincidiu com a época das chuvas.

A análise microscópica das gônodas dos machos de $M$. molossus permitiu separar dois grupos de indivíduos. Um, com espermatozoides no epidídimo, isto é, sexualmente ativo, e outro, sem espermatozoides no epidídimo. A caracterização da atividade sexual dos machos foi confirmada pelo estudo comparativo das medidas de comprimento dos testículos dos dois grupos. Verificou-se assim, que há diferenças estatisticamente significativas, a um nível de significância de $1 \%$, sendo os testículos dos machos sexualmente ativos maiores $(x=5,58 \mathrm{~mm})$ do que os dos sexualmente inativos $(\mathrm{x}=3,25 \mathrm{~mm})$.

Machos sexualmente ativos foram encontrados ao longo de quase todo o ano, o que sugere que, após o amadurecimento sexual, armazenam espermatozoides mesmo fora de época de acasalamento (Fig. 6).

\section{DISCUSSÃO}

A assimetria morfologica e funcional das gônodas de fêmeas de Molossus molossus aqui observadas têm sido encontrada em diversas espécies de quirópteros. KRUTZSCH \& CRICHTON (1985) estudando Molossus fortis verificaram que esta espécie apresenta não só assimetria entre os cornos uterinos e ovário direito e esquerdo, como também ausência de estágios avançados de desenvolvimento folicular e conseqüente falta de produção de óvulos no ovário esquerdo. $\mathrm{O}$ mesmo foi verificado em Rhinolophus hipposideros por MUTERE (1967). Segundo HOOD \& SMITH (1983) a unilateralidade de funçã̃ do útero e ovário ocorre também no gênero Noctilio e em diversas espécies de Phyllostomidae.

$\mathrm{O}$ fato de apenas um ovário manter-se ativo é ainda assunto que merece maiores estudos. Segundo JERRETT (1979) mecanismos como vascularidade assimétrica e presença ou ausência de receptöres apropriados não foram suficientemente estudados para elucidar a dominância ovariana que ocorre em muitos quirópteros. Por outro lado, BLEIER \& EHTESHAMI (1981) demonstraram que, após a remoção cirúrgica do ovário direito em Macrotus californicus, o ovário esquerdo tornou-se ativo, passando a ovular com a subseqüente implantação do blastocisto no corno esquerdo do útero.

M. molossus desenvolve apenas um filhote por gestação, apresentando dois períodos reprodutivos ao longo do ano, março-abril e novembro, nas populações observadas. MARQUES (1986) trabalhando em Manaus, AM, encontrou fêmeas 


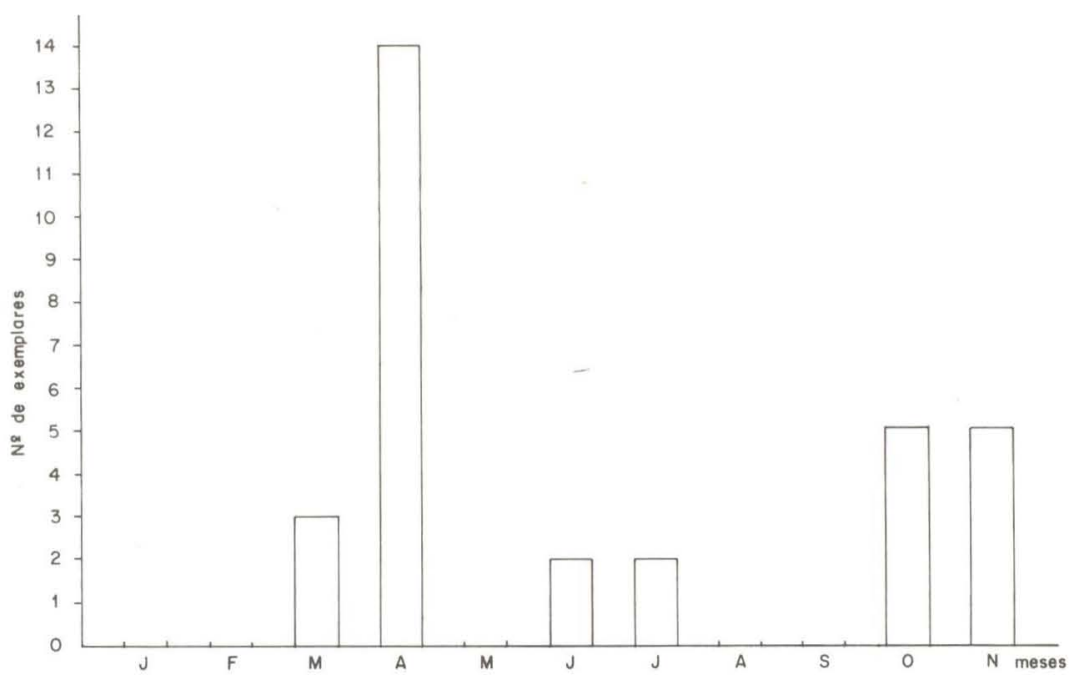

Fig. 6. Relação entre o número de machos de Molossus molossus com espermatoz6ides no epidídimo e os meses do ano.

grávidas de Molossus ater em quase todos os meses do ano, caracterizando-as como poliestras.Este fato indicaria que não há relação entre os períodos reprodutivos e as estações do ano em regiões onde a temperatura se mantém mais ou menos constante e os períodos de chuva e seca são bem definidos.

KRUTZSCH \& CRICHTON (1985) citam a criação em cativeiro de $M$. molossus e M. ater onde se observou que, aparentemente, as fêmeas que participam de um período reprodutivo não o fazem no período subseqüente sendo, na realidade, indivíduos monoestros. Assim, haveria uma alternância de fêmeas grávidas dentro da mesma população.

Os dados parecem demonstrar que há necessidade de maiores estudos para uma melhor interpretação dos fenômenos fisiológicos da reprodução associados às condições ambientais e à disponibilidade de alimento.

MARQUES (1986) classifica os machos de $M$. ater como ativos quando o comprimento do testículo é maior ou igual a $5,5 \mathrm{~mm} \mathrm{e}$, inativos, quando o testículo apresentar comprimento menor. No presente trabalho também se observaram diferenças estatisticamente significativas no tamanho dos testículos dos machos sexualmente ativos e inativos.

Segundo BRONSON (1985) os machos de mamíferos podem permanecer ativos reprodutivamente fora das épocas em que as fêmeas se encontram receptivas. Mesmo em situações de forte sazonalidade, em que é energeticamente impossível para as fêmeas se reproduzirem durante uma parte do ano, os machos podem entrar em atividade reprodutiva antes das fêmeas e sair desta fase após elas. Em outros casos, o controle ambiental sobre a reprodução pode atuar diferentemente, qualita- 
tiva e quantitativamente nos dois sexos. Isto explicaria as diferentes estratégias observadas entre $M$. molossus no presente estudo e $M$. ater na Amazônia pois, segundo MARQUES op. cit., nesta última espécie a atividade reprodutiva dos machos pareceria estimulada pela ocorrência de um maior número de fêmeas receptivas, o que não seria o caso dos exemplares de $M$. molossus aqui estudados.

\section{AGRADECIMENTOS}

Agradecemos à prof ${ }^{\text {a }}$ Sônia Maria Lauer de Garcia, do Departamento de Ciências Morfológicas da UFRGS, por ter propiciado treinamento de técnicas histológicas; ao Dr. Joaquim Eduardo Alencar, Coordenador do Núcleo de Medicina Tropical da Universidade Federal do Ceará e à sua equipe, os quais facilitaram a realização deste trabalho e à SUCAM, pelo auxílio no trabalho de campo.

\section{REFERÊNCIAS}

BLEIER, W.J. \& M. EHTESHAMI 1981. Ovulation following unilateral ovariectomy in the California leaf-nosed bat (Macrotus californicus). J Reprod. Fert., Oxford, 63: 181-183.

BORNE, B. 1985. Ecologia de quirópteros da Estação Ecológica do Taim, com ênfase na família Molossidae $88 \mathrm{f}$. Tese (Mestrado-Ecologia) UFRGS. Porto Alegre, 1985. Não publicada.

BRONSON, F.H. 1985. Mammalian reproduction: an ecological perspective. Biol. Reprod , 32: $1-26$.

CARTER, D.C. 1970. Chiropteran Reproduction. In: Slaughter. B.H. \& WALTON, D.W. ed. About Bats: A chiropteran biology symposium. Dallas, Southern Methodist University Press, p. 233-246.

FLEMING, T.H.; E.T. HOOPER \& D.E. WILSON 1972. Three Central American Bat Communities: Structure, Reproductive Cicles and Movement Patterns. Ecology, Brooklyn, 53(4): 555-569.

HOOD, C.S. \& J.D. SMITH 1983. Histomorphology of the female reproductive tract in Phyllosmotomoid bats. Occ. Pap. Mus. Texas Tech.Univ., Lubbock (86):1-38.

JERRET, D.P. 1979. Female reproductive patterns in non-hibernating bats. J. Reprod. Fert., Oxford, 56: 369-378.

KRUTZSCH, P.H. \& E.G. CRICHTON 1985. Observations on the reproductive cycle of female Molossus fortis (Chiroptera: Molossidae) in Puerto Rico. J. Zool., London, 207 (A):137-150.

MARQUES, S.A. 1986. Activity cycle, feeding and reproduction of Molossus ater (Chiroptera: Molossidae) in Brazil. Bolm Mus. para. Emilio Goeldi, Belém, Ser. Zool., 2(2): 159-179.

MUTERE, F.A. 1967. The breeding biology of equatorial vertebrates: in the fruit bat Eidolon helvum, at latitude $0^{\circ} 20^{\prime} \mathrm{N}$. J, Zool. London, 153: 153-161.

PERACHI, A.L. 1968. Sobre os hábitos de Histiotus velatus (Geoffroy, 1824) (Chiroptera, Vespertilionidae). Revta bras. Biol. Rio de Janeiro, 28(4): 469-473. 
RACEY, P.A. 1982. Ecology of bat reproduction. In: Kunz, T.H. ed. Ecology of bats New York, Plenum Press, p. 57-104.

SANTOS, A.B. 1978. Identificação e dados bio-ecológicos dos quirópteros do vale do Rio dos Sinos e arredores. Estudos Leopoldensis, São Leopoldo, 13(45): 75-126.

SNEDECOR, G.W. \& W.G. COCHRAN 1967. Statistical Methods. Iowa State Univ. Press, Ames. 593 p.

TADDEI, V.A. 1976. The reproduction of some Phyllostomidae (Chiroptera) from the Northwestern region of the State of Säo Paulo. Bolm Zool., Univ. São Paulo (1). 1-18.

TADDEI, V.A. 1980. Biologia reprodutiva de Chiroptera: perspectivas e problemas. Inter-Facies, São José do Rio Preto, (6): 1-18.

TADDEI, V.A.; L.D. VIZOTTO; S.M. MARTINS 1976. Notas Taxonomicas e biológicas sobre Molossops brachymeles cerastes (Thomas, 1901) (Chiroptera, Molossidae). Naturalia, Lisboa, 2: 61-69.

WILLIG, M.R. 1983. Composition, microgeographic variation, and sexual dimorphism in Caatingas and Cerrado bat communities from northeast Brazil. Bull, Carnegie Mus. Nat. Hist, Pittsburg, (23):1-131, 8 figs., 29 tab.

WILLIG, M.R. 1985 a. Ecology, reproductive biology and systematics of Neoplatymops mattogrossensis (Chiroptera, Molossidae). J. Mammal., Lawrence, 66(4): 618-628.

WILLIG, M.R. 1985b. Reproductive patterns of bats from Caatingas and Cerrado biomes in Northeast Brazil. J. Mammal, Lawrence 66(4): 668-681.

WILSON, D.E. 1979. Reproductive patterns. In: BAKER R.J.; JONES JR. J.K.; CARTER, D.C. eds. Biology of Bats of the New World family Phyllostomidae. Part III. Spec. Publ. Mus. Texas Tech.Univ. 16: 317-318. 